\title{
Alterstice
}

Revue internationale de la recherche interculturelle

International Journal of Intercultural Research

Revista International de la Investigacion Intercultural

\section{L'interculturel au Québec : rencontres historiques et enjeux politiques, sous la direction de Lomomba Emongo et Bob W. White}

\section{Jade Boivin}

Volume 5, numéro 2, 2015

URI : https://id.erudit.org/iderudit/1036697ar

DOI : https://doi.org/10.7202/1036697ar

Aller au sommaire du numéro

Éditeur(s)

Alterstice

ISSN

1923-919X (numérique)

Découvrir la revue

Citer ce compte rendu

Boivin, J. (2015). Compte rendu de [L'interculturel au Québec : rencontres

historiques et enjeux politiques, sous la direction de Lomomba Emongo et Bob

W. White]. Alterstice, 5(2), 123-129. https://doi.org/10.7202/1036697ar 


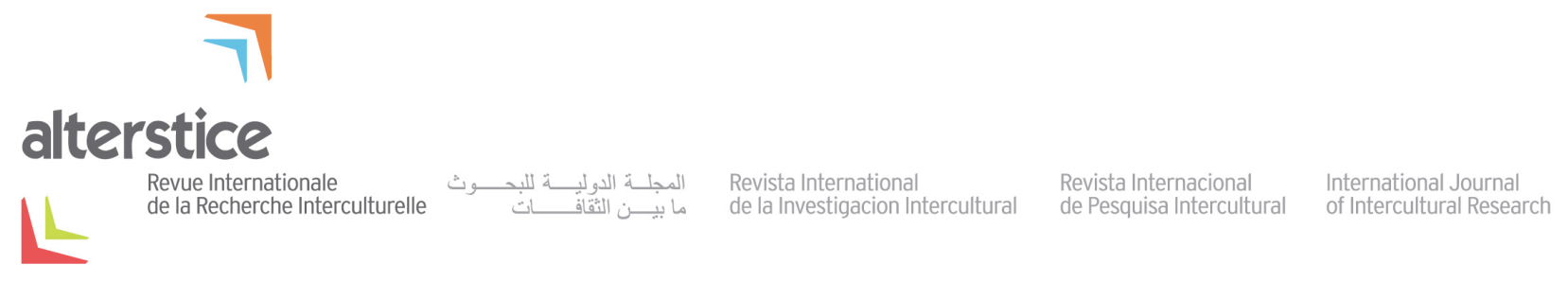

NOTE DE LECTURE

\title{
L'interculturel au Québec : rencontres historiques et enjeux politiques, sous la direction de Lomomba Emongo et Bob W. White
}

Jade Boivin ${ }^{1}$

\author{
Rattachement de l'auteure \\ 1 Université du Québec à Montréal et Groupe d'étude et de recherche axées sur la communication internationale \\ et interculturelle, Montréal, Canada
}

\section{Correspondance}

jade.boivin@gmail.com

\section{Références de l'ouvrage}

Emongo, L. et B. W. White (dir.) (2014). L'interculturel au Québec : rencontres historiques et enjeux politiques. Montréal : Presses de l'Université de Montréal.

\section{Pour citer cet article}

Boivin, J. (2016). L'interculturel au Québec : rencontres historiques et enjeux politiques [Note de lecture]. Alterstice, 5(2), 123-129.

\section{Introduction}

Ce livre est le résultat d'un projet de recherche mené par le Laboratoire de recherche en relations interculturelles (LABRRI) qui porte sur les dynamiques interculturelles dans une optique relationnelle au sein des villes interculturelles. La conjoncture de cinq événements qui se sont déroulés au Québec est à l'origine de cet ouvrage : la Commission Bouchard-Taylor, la fermeture de l'Institut interculturel de Montréal, la nomination de Montréal comme cité interculturelle en Amérique du Nord et la parution du livre de Gérard Bouchard, L'interculturalisme, en 2012. Les auteurs jugent qu'à la lumière de cette chronologie d'événements, il est nécessaire de se pencher sur l'évolution et sur l'état de l'interculturel et de l'interculturalisme au Québec. Le livre regroupe les chapitres en trois parties: l'interculturalisme au Québec, l'archéologie d'une terminologie et finalement l'interculturel au-delà du Québec. D'entrée de jeu, ils expliquent qu'une méfiance à propos de la compréhension et de l'utilisation de l'interculturalisme constitue une motivation à la création de ce livre. Les auteurs sont d'avis que l'intérêt porté à la question large de l'immigration est empreint d'une contradiction. Ainsi, le livre explore l'essence du terme interculturalisme, à savoir s'il est une "réelle idéologie politique " ou bien si le terme n’a pas été récupéré à des fins d'instrumentalisation politique. De plus, dans un contexte où la question de l'identité nationale et l'aspect francophone de sa culture octroient au Québec une place particulière en Amérique du Nord, les auteurs sont d'avis 
que le modèle de l'interculturalisme québécois s'inscrit en opposition au multiculturalisme canadien, comme une matérialisation de l’opposition entre le Québec francophone et le Canada anglophone.

\section{Première partie : De l'interculturalisme québécois}

\section{Chapitre 1 - Quel métier pour l'interculturalisme au Québec? (Bob W. White)}

Le premier chapitre s'intéresse aux différentes définitions de l’interculturalisme qui ont été discutées dans le cadre du symposium " Interculturalisme 2011 » qui a eu lieu à la Bibliothèque nationale du Québec en avril 2011. grande diversité de définitions a émergé d'une des tables rondes du symposium. L'auteur avance qu'il n'y a pas un réel accord sur ce qu'est l'interculturalisme, et ce, même au sein des spécialistes universitaires et des intervenants sur le terrain. II critique la position de Bouchard qui a proclamé, après la tenue de la Commission Bouchard-Taylor, que les Québécois étaient généralement en accord avec l'interculturalisme. Ainsi, l'auteur conteste la validité scientifique des résultats obtenus dans le cadre de la consultation publique de la Commission. Selon lui, il n'y a aucune preuve que la participation à cette consultation était représentative de l'opinion des citoyens québécois. De plus, comme aucun outil n'a été utilisé afin de mesurer si les participants partageaient la même définition de l'interculturalisme, il est impossible de dire que l'ensemble des participants à la consultation était en accord et encore moins de généraliser cet accord. Dans la deuxième partie du chapitre, l'auteur mène une analyse discursive des documents gouvernementaux et universitaires portant sur l'interculturalisme au Québec. Cette analyse a pour but d'identifier les principaux éléments tous idéologiques ainsi que les « angles morts » de l'interculturalisme. Puis, dans la dernière partie du chapitre, il se penche sur les trois registres de l'interculturel au Québec. II est question de l'interculturel en tant que réalité sociologique, c'est-à-dire d'espace de rencontre interculturelle entre deux personnes d'appartenances culturelles différentes, ensuite comme un concept-mythe ou idéologie politique qui s'oppose au multiculturalisme et finalement à titre d'orientation épistémologique empreinte d'une considération pour l'Autre. White s'intéresse aux effets nuisibles de certaines définitions de l'interculturel sur le tissu social québécois. D’un même souffle, il explore le glissement sémantique du terme interculturalité vers interculturalisme. En se basant sur cette prémisse, l'auteur formule une critique où il accuse l'interculturalisme d'être devenu un instrument politique plutôt qu'un réel modèle d'intégration. Ainsi, il est reproché à Bouchard de prescrire l'interculturel tout en prétendant le décrire. Finalement, l'auteur aborde le débat politique particulièrement polarisant qui oppose le multiculturalisme et l'interculturalisme. Il est d'avis que de préférer le modèle de l'interculturalisme au modèle du multiculturalisme comme une solution au vivre ensemble parce qu'il respecte l'aspect "distinct » de la société québécoise est une erreur. Selon lui, l'interculturalité québécoise ne découle pas des politiques étatiques basées sur l'interculturalisme. Du même souffle, il incite le lecteur à contester le rejet du multiculturalisme comme modèle d'intégration pour le Québec.

\section{Chapitre 2 -L'interculturalisme et la Commission Bouchard-Taylor (Jorge Frozzini)}

Le deuxième chapitre du livre porte sur l'historique de l'interculturalisme en tant que modèle québécois. Selon son analyse du contexte sociopolitique entourant la Commission Bouchard-Taylor, Jorge Frozzini est d'avis que le processus de consultation publique n'a pas été neutre. En effet, il avance que celui-ci était biaisé par les Documents de consultations qui privilégient le modèle d'interculturalisme, et ce, de plusieurs manières. De plus, Frozzini estime que des orientations politiques subtiles ont guidé le processus, notamment en présentant l'interculturalisme et le multiculturalisme dans un paradigme de dualité. Le multiculturalisme a été présenté comme un modèle indésirable pour le Québec, car il constituerait une menace à la survivance de la langue française et mettrait en péril les valeurs " distinctes » québécoises. L'auteur est d'avis que ceci a eu pour effet de nuire au ciment social qui unit les différentes composantes interculturelles québécoises. II explique que l'interculturalisme, comme modèle d'intégration de l'immigration, considère celle-ci comme une entité monolithique. Ainsi, les particularités des différents groupes sont ignorées et il est attendu que les différentes communautés s'intègrent à la société québécoise sans changer cette dernière, ce qui est en opposition au multiculturalisme canadien. Frozzini conclut en expliquant que la question identitaire des Québécois francophones trouve un écho dans le modèle de l'interculturalisme. Tout en reconnaissant l'importance de la Commission Bouchard-Taylor, ce chapitre expose les différents biais à considérer, surtout concernant les conclusions de la Commission au sujet de l'interculturalisme comme meilleur modèle pour le Québec.

Alterstice - Revue Internationale de la Recherche Interculturelle, vol. $5, n^{\circ} 2$ 


\section{Chapitre 3 -La mise en œuvre des recommandations de la Commission Bouchard-Taylor (François Rocher)}

Ce troisième chapitre rapporte une étude menée par François Rocher dont le sujet était le suivi des 37 recommandations de la Commission, celles-ci étant divisées à l'origine en cinq axes. L'auteur est d'avis que ces axes ne permettent pas de conceptualiser les différentes dimensions de la diversité. Ce chapitre présente donc la méthodologie qui a permis une analyse différente de celle réalisée par le ministère de l'Immigration et des Communautés culturelles, qui déclarait que $80 \%$ des recommandations établies par la Commission BouchardTaylor avaient été suivies. François Rocher est d'avis que l'impact de la Commission a été gonflé par le gouvernement. Divisé en deux parties, le chapitre présente, dans un premier temps, des éléments théoriques, alors que la seconde partie propose les résultats et les conclusions de l'étude qui explore le suivi concernant les recommandations de la Commission. Tout d'abord, une typologie est proposée afin de définir de manière exhaustive l'interculturalisme. Pour ce faire, Rocher a utilisé la grille d'analyse de Salée (2010) qui est divisée en trois axes ou dimensions: instrumentale, humaniste et citoyenne. La dimension instrumentale considère les échanges interculturels comme des occasions de contrer les rapports de pouvoir et les inégalités dans une perspective d'autonomisation par le biais de l'insertion sur le marché du travail. La dimension humaniste fait appel à l'aspect du savoir-être dans un contexte de pluricité ethnique. Cet aspect valorise l'expérience interculturelle et vise à ce que celle-ci se déroule dans le respect de la diversité et le sentiment de sa reconnaissance. La dimension citoyenne a pour but de créer un cadre commun partagé afin de préserver une cohésion sociale et prévenir la ghettoïsation. Rocher est d'avis que cette grille est pertinente pour deux raisons. Premièrement, elle permet de vérifier si le travail accompli dans le cadre de la Commission a privilégié certains aspects des politiques de gestion de la diversité en défaveur des autres. La grille est aussi utilisée afin d'analyser le suivi des autorités publiques quant aux recommandations émises par la Commission. L'analyse a été menée de manière systématique en attribuant une pondération à l'ensemble des recommandations puis en les classifiant selon les trois axes d'analyse. Par la suite, une vérification a été faite pour savoir s'il y avait eu un suivi et une politique mise en place pour chacune des recommandations. Les conclusions de cette analyse démontrent que $80 \%$ des recommandations ont eu un suivi, mais qu'il est faux d'affirmer que le gouvernement a mis en place ce même pourcentage de politiques concernant les recommandations. Les résultats de l'analyse démontrent aussi qu'une majorité de ces recommandations appartenaient à la dimension humaniste, tandis que la dimension citoyenne a constitué moins d'un quart des recommandations.

\section{Chapitre 4 - L'interculturalisme selon Gérard Bouchard (Jorge Frozzini)}

Ce chapitre est une analyse critique de trois écrits de Gérard Bouchard dans une perspective évolutive de sa réflexion et de sa définition de l'interculturalisme. Frozzini cherche à mieux comprendre comment la conception de I'interculturalisme défendue par Bouchard est devenue une référence en la matière. II cherche aussi à en mesurer les conséquences sur les rapports d'altérité au sein de la société québécoise. Le chapitre est divisé en deux: l'auteur présente d'abord l'essentiel de chacun des trois textes signés par Bouchard, puis en fait l'analyse. Le premier texte dont il est question est "Qu'est-ce que l'interculturalisme?». À son propos, on souligne que Bouchard insiste sur la protection de la majorité francophone dans la gestion de la pluricité. Dans le deuxième texte, L'interculturalisme québécois. Esquisse d'un modèle, Bouchard offre une définition de l'interculturalisme qui se clarifie dans le troisième texte choisi, soit L'interculturalisme. Selon Frozzini, Bouchard définit toujours l'interculturalisme selon un paradigme de dualité et selon sa propre dimension microsociale personnelle et donc subjective. II affirme, dans la deuxième partie du chapitre, que Bouchard instrumentalise à des fins politiques le modèle de l'interculturalisme. Il est d'avis que la définition de l'interculturalisme de Bouchard perpétue un rapport de force entre majorité francophone et minorité immigrante au sein de l'espace public, notamment en biaisant la représentation d'un réel dialogue interculturel. L'argumentaire de Frozzini à ce sujet s'articule en trois points. Tout d'abord, la gestion de la diversité selon Bouchard est considérée comme un défi que les Québécois « de souche " sont responsables de relever. La critique adressée à cette vision de l'immigration est que la représentation monolithique des immigrants ainsi que leur représentation en tant qu'entité passive doivent être gérées par les citoyens québécois "de souche". Le deuxième argument est l'effet négatif de la préséance de la majorité francophone par rapport aux autres communautés ainsi que sa protection. Ces effets se font sentir sur le tissu social québécois et ont pour effet de renforcer le sentiment d'altérité entre Québécois et immigrants. Le troisième 
argument est celui de l'instrumentalisation et de l'effacement des minorités selon une perspective utilitariste afin d'assurer la survivance de la langue française au Québec.

\section{Deuxième partie : l'archéologie d'une terminologie}

Chapitre 5 - Le congrès juif canadien et la promotion de l'éducation interculturelle (1947-1975) (Pierre Anctil)

Ce chapitre, qui amorce la deuxième partie du livre, porte sur l'influence de la communauté juive de Montréal, plus précisément de l'organisme du congrès juif canadien (CJC) sur les politiques publiques en lien avec l'approche interculturelle. Le congrès juif canadien (CJC) est un espace démocratique où les membres de la communauté juive provenant d'horizons politiques et culturels différents peuvent échanger à propos de la place de la communauté au sein de la société québécoise et canadienne. L'institution se penche sur la question de l'intégration de la communauté juive tout en cherchant à préserver l'autonomie de la communauté. Ce chapitre s'intéresse au travail précurseur du CJC en matière d'éducation interculturelle ainsi qu'à ses initiatives concrètes en ce sens. Il aborde de plus le rôle des différents partenaires internationaux du CJC et leur influence sur leurs activités pédagogiques en matière de rapprochement interculturel. II est ensuite question de la contribution d'Arthur Lermer, intellectuel progressiste de gauche de confession juive, aux réflexions du CJC quant aux politiques publiques canadiennes en matière de pluralisme culturel. Les réflexions analysées par Anctil relatent que Lemer, appuyé par la mission du CJC, a mené un travail considérable de sensibilisation à propos de l'éducation interculturelle auprès de multiples instances décisionnelles gouvernementales et communautaires en éducation interculturelle, dans le but de combattre les préjugés de la population à propos du pluralisme religieux. L'auteur souligne que les liens entretenus par l'institution étaient surtout envers la communauté canadienne et anglophone québécoise. Ainsi, ses réflexions sont inspirées du modèle d'intégration d'immigration américain. Ce modèle est basé sur la prémisse que les relations interculturelles entre immigrants et citoyens américains sont une opportunité de créer une nouvelle culture politique. Dans la troisième partie du texte, il est question de l'apport du Bureau of Intercultural Education, fondé en 1934 dans la ville de New-York par Rachel Dubois, une enseignante et militante antiraciste. C'est dans le travail de madame Dubois qu'émerge la notion d'interculturel, en opposition à la pensée assimilationniste qui prévalait à l'époque. L'auteur termine le texte en invitant le gouvernement québécois à reconnaître l'origine du modèle de l'interculturalisme. Du même souffle, il critique le manque de reconnaissance de la minorité juive par rapport à l'éducation interculturelle et pour son influence sur les politiques publiques canadiennes et québécoises en matière d'intégration de l'immigration et du vivre-ensemble.

\section{Chapitre 6 - Panikkar, l'interculturalisme au Québec (Lomomba Emongo)}

Ce chapitre porte sur l'apport des travaux de Raimon Panikkar à travers son implication au sein de l'Institut interculturel de Montréal (IIM) dès 1968. Au cours de sa carrière, Panikkar a été professeur à l'université de Harvard et à l'Université de Californie à Santa Barbara. II s'est intéressé au pluralisme religieux dans le cadre de la relation, qu'il qualifie d'hégémonique, entre Occident et Orient. Polyglotte, se disant à la fois bouddhiste, chrétien et hindou et jouissant de la double nationalité espagnole et indienne, Panikkar a défendu trois thèses de doctorat, en théologie, en sciences et en philosophie. L'œuvre de Panikkar est pertinente notamment à cause de sa démarche ainsi que de sa posture épistémologique par rapport à l'interculturalisme. La quête de réconciliation de ses multiples identités religieuses amène Panikkar à s'intéresser au dialogue religieux. C'est dans le cadre de ce cheminement qu'il sera amené à travailler avec Robert Vachon, directeur de l'IIM. Emongo est d'avis que la composition identitaire diversifiée de Panikkar est particulièrement pertinente car elle est un « signe annonciateur d'une dimension humaine de notre temps » (p. 140). Panikkar dédiera 70 ans de sa vie à écrire. Il sera toujours à la fois objet et sujet de la recherche de dialogue qui anime ses multiples œuvres. D'ailleurs, sa double nationalité à la fois orientale et occidentale sera l'élément déclencheur de sa réflexion à propos du dialogue interculturel. L'auteur nous introduit ensuite à la pensée de Panikkar, notamment en expliquant l'herméneutique diatopique, c'est-à-dire une herméneutique inclusive d'une pluralité de traditions et qui s'intéresse à l'herméneutique à la fois essentielle et existentielle. Dans la pensée de Panikkar, le caractère inclusif et pluriel de cette herméneutique lui permet « un dépassement des distances " entre les différentes religions par le concept de dialogue dialogique. Le dialogue dialogique est un espace de rencontre qui mise davantage sur les ressemblances entre les différentes religions et permet de "transcender la distance » entre celles-ci. Ceci permet de tendre vers une conception plus universelle 
de la religion, ce que désigne la notion de pluralisme originel. L'auteur s'intéresse par la suite à l'interrelation entre religion et culture ainsi qu'au dialogue interreglieux et au dialogue interculturel dans l'œuvre de Panikkar. Finalement, Emongo se base sur l'œuvre de Panikkar afin d'expliciter la définition de l'interculturalisme selon ce dernier : un concept qui permet d'explorer la richesse de notre propre culture et de l'autre. Selon Panikkar, l'interculturalisme permet une conception plus universelle de I'humanité. Son influence au sein de I'IIM est brièvement abordée. Elle jouera un rôle déterminant dans l'endossement de l'interculturalisme par l'institution.

\section{Troisième partie : l'interculturel en action}

\section{Chapitre 7 - Quelques figures marquantes des débuts de l'interculturel au Québec (Joseph Josy Lévy)}

Ce chapitre, qui amorce la troisième partie du livre, s'intéresse à l'évolution de l'interculturel au Québec, plus précisément à l'apport du Centre Mochanin et de l'Institut interculturel de Montréal. Lévy juge pertinent de s'y attarder car l'historique de ces institutions met en évidence plusieurs dimensions de l'interculturel au Québec. Le chapitre présente l'apport de deux pionniers de l'interculturel, Robert Vachon et Jacques Langlais, tous deux prêtres catholiques romains s'intéressant à l'approche spirituelle de traditions méditatives indiennes. L'auteur se base sur l'autobiographie de Jacques Langlais, prêtre de la Congrégation de Sainte-Croix, fondateur et ancien directeur du Centre Mochanin, centre qui a eu une influence considérable sur l'étude de l'interculturel au Québec. La création du centre Mochanin, intimement liée à l'existence du Carrefour international des étudiants, dont la mission a été d'accueillir, entre 1961 et 1967, des étudiants et des stagiaires du Québec et de l'étranger ainsi que des immigrants, dans le but de favoriser les échanges interculturels et d'offrir un espace de dialogue informel où il serait possible de débattre des différentes manières de penser. Le centre Mochanin, nommé ainsi en l'honneur du père Mochanin qui a œuvré pour la rencontre interculturelle, est fondé en 1963. Josy Lévy explique que, selon Jacques Langlais, la décentration ainsi que la notion de convivance sont à la base de la rencontre interculturelle. Ces valeurs étaient à la base des seminars œcuméniques qu'organisait Langlais. D'inspiration rogérienne, ils étaient basés sur la communication interpersonnelle au sein du dialogue interculturel. L'écoute et le dialogue y étaient privilégiés. Ainsi, concrètement, des groupes restreints de 6 à 7 personnes étaient formés, puis guidés par un animateur et les membres du groupe abordaient des thématiques en lien avec l'interculturel. Les discussions étaient menées dans une optique existentielle. Le succès de ces seminars a engendré le développement d'autres seminars portant sur les grandes religions. Ainsi, Lévy souhaite démontrer que, dès son émergence au Québec, l'interculturel s'articulait autour du pluralisme religieux. Puis l'arrivée de Robert Vachon comme codirecteur en 1966 a amené un changement d'orientation plus théorique et critique des activités du centre. Suite à une réflexion à propos d'une redéfinition de la mission du Centre Mochanin, celui-ci devient en 1990 le Centre interculturel de Montréal. Sa mission devint dès lors laïque, axée sur l'intellect et davantage enracinée dans la réalité interculturelle locale. L'auteur conclut en affirmant que le caractère religieux des écrits du Centre Mochanin et de l'IIM est peutêtre dépassé, mais que les approches critiques et méthodologiques expérientielles demeurent encore aujourd'hui pertinentes.

\section{Chapitre 8 -Le destin d'une terminologie, de l'interculturel à l'interculturalisme (Danielle Gratton)}

Ce chapitre porte sur les politiques d'accessibilité des services sociaux pour les personnes issues de minorités culturelles en lien avec les travaux de l'IIM. L'auteure, Danielle Gratton, utilise un entretien mené avec madame Kalpanas Das, qui a été la dernière directrice de l'IIM, afin de comprendre comment la notion d'interculturel a été véhiculée dans la société québécoise et auprès des institutions publiques. Au cours de l'entretien, la question de l'évolution de la sémantique du mot interculturalisme au Québec est aussi abordée. D'entrée de jeu, l'auteure différencie deux termes : I'interculturel tel qu'il a été conceptualisé à l'IIM et l'interculturalisme comme modèle politique au Québec. Le chapitre se divise en trois thèmes, selon les questions adressées à Das. La première concerne l'évolution et la pratique interculturelle à l'IIM. Lors de sa fondation en 1963, le Centre Mochanin est dirigé par Langlais qui est animé par un désir d'éducation du peuple québécois en matière de pluralisme religieux. Sous la direction de Vachon dès 1967, la mission du Centre change et vise davantage à favoriser les rencontres et le dialogue interculturels. Vachon et Das travailleront ensemble dès 1971 et aspireront à dépasser les frontières qui existent entre la culture et la religion au sein de l'imaginaire occidental. Madame Das contribuera aux activités de I'IIM en établissant un programme d'échange interculturel qui s'adressent aux adultes. La deuxième question porte sur la diffusion de la pratique interculturelle auprès de différentes institutions publiques du Québec. En réponse à 
cette question, Gratton fait un survol des besoins grandissants des membres des communautés issues de l'immigration par rapport aux services sociaux publics québécois. On y apprend ainsi davantage à propos du travail de formation accompli par l'IIM en matière d'interculturalité auprès des employés de la fonction publique. Les formations en sensibilisation interculturelle offerte dans le milieu de l'éducation de l'immigration et de la santé ont aussi joué un rôle clef dans le rayonnement de la vision de l'interculturel de l'IIM. Finalement, la troisième question aborde le " glissement » du sens du mot interculturel. Das est en effet d'avis qu'il y a un " glissement » du sens du mot interculturel de la manière dont l'entendait l'IIM dans la notion d'« interculturalisme ». Ainsi, l'interculturalisme comme modèle d'intégration de l'immigration, qui serait l'institutionnalisation de l'interculturalité, ne respecterait pas l'essence de ce terme. La prévalence de l'aspect citoyen de l'identité sur l'aspect culturel serait à l'origine de ce changement sémantique.

\section{Chapitre 9 - La notion laïque, l'importance de la langue dans la vie des nations (Charles Blattberg)}

Le sujet de ce chapitre porte sur la question des accommodements raisonnables. L'auteur fait valoir qu'il est nécessaire d'aller au-delà des accommodements et de la négociation entre groupes majoritaires et minoritaires. II reconnaît l'importance et l'inexorabilité de la négociation. Charles Blattberg est d'avis qu'il est nécessaire de savoir et de comprendre avant d'entreprendre toute négociation. Le chapitre explore l'apport des créations artistiques à la construction des nations, qui selon l'auteur ont joué un rôle primordial dans l'émergence de celles-ci. II poursuit en définissant les notions de création et d'interprétation, à la base de l'argumentaire présenté dans le texte. Ainsi, les interprétations permettent de donner un sens à une situation alors que les créations sont des " inspirations inspirées » qui sont à la fois interprétatives et d'ordre pratique. L'auteur déveloape son idée en abordant la pensée de différents auteurs modernes et romantiques au sujet de la nation. II est d'avis que la création est à l'origine de l'émergence des nations en ce sens qu'elle est productrice du mythe fondateur qui est à l'origine du sentiment nationaliste. C'est ainsi qu'il explique que la question des accommodements raisonnables au Québec est intrinsèquement liée à ces notions de création et d'interprétation, permettant un partage qui mène à une réconciliation entre les différents groupes qui compose la nation.

\section{Chapitre 10 - Introduction à une épistémologie de l'inter-cultures (Lomomba Emongo)}

Ce chapitre est une introduction à une recherche épistémologique de l'interculturel. L'argumentaire est basé sur diverses études ayant porté sur l'interculturalisme québécois. Le premier texte utilisé s'intitule Les fondements théoriques de l'interculturalisme (CRIEC, 2011) et le deuxième texte est Qu'est-ce que l'interculturalisme? (Bouchard, 2011). L'auteur fait ressortir divers aspects de la question épistémologique de l'interculturel. Plus spécifiquement, il dégage trois points d'intersection de l'analyse de ces textes : l'interculturalisme s'inscrit dans une logique pragmatique, il est considéré comme un fait tangible et il sert le groupe majoritaire. Outre ces constatations, Emongo est d'avis qu'il y a un flou à la définition de l'interculturalisme d'un point de vue épistémologique. Ayant pour but d'entamer une réflexion offrant le début d'une réponse, il se penche sur la composition du mot interculturalisme. Ainsi, il s'intéresse aux potentialités syntaxiques et sémantiques du mot " inter-culture », terme composé de trois éléments : le préfixe inter, le trait d'union et le suffixe " culture ». Les conclusions de cette réflexion l'amènent à penser que le terme évoque la rencontre de cultures ainsi que le rapport d'altérité. II présente ces conclusions sous trois lieux qui se veulent une introduction à l'épistémologie de l'interculturalisme.

\section{Critique}

Ce livre s'adresse à des lecteurs familiers des différents travaux et réflexions universitaires entourant l'interculturalisme québécois. De l'introduction jusqu'au dernier chapitre, il est question du travail de Gérard Bouchard, qui s'y trouve fortement critiqué. Il est donc important de connaître l'œuvre et les prises de position de Bouchard afin de bien saisir l'ensemble des éléments mentionnés dans cet ouvrage, qui se présente comme un dialogue sur l'interculturalisme. Ce regard critique sur le discours dominant entourant l'interculturalisme québécois est rafraîchissant. Les différents auteurs offrent à la fois des pistes de réflexion nouvelles tout en complétant des critiques et des questions déjà formulées à l'égard du modèle d’intégration québécois. 
Par contre, il aurait été intéressant de suggérer des pistes de réflexion afin de remplacer ou d'améliorer le modèle d'interculturalisme fortement critiqué dans le cadre du livre. Concernant la fluidité de la lecture, le fil conducteur entre les différents chapitres n'est pas toujours évident. Dans cette optique, il est préférable pour le lecteur d'appréhender ce livre selon les chapitres qui l'interpellent plutôt que de le voir comme un tout. II est important de mentionner que chacun des chapitres est pertinent et intéressant à sa manière. Ceci dit, le niveau de langage, la structure des chapitres et le style littéraire varient grandement d'un texte à l'autre, ce qui peut rendre la lecture ardue. Bien entendu, ceci est sans doute normal dans un ouvrage collectif. Bref, tous lecteurs intéressés par le sujet sauront trouver leur compte grâce à la diversité des aspects de l'interculturalisme présentés ainsi que l'originalité des points de vue. 